Proceedings of the 1997 IEEE International Conference on Robotics and Automation Albuquerque, New Mexico - April 1997
C1997 IEEE. Personal use of this material is permitted. However, permission to reprint/republish this material for advertising or promotional purposes or for creating new collective works for resale or redistribution to servers or lists, or to reuse any copyrighted component of this work in other works must be obtained from the IEEE.

\title{
Controller for a High Strain Shape Memory Alloy Actuator: Quenching of Limit Cycles
}

\author{
Danny Grant \\ Vincent Hayward \\ Department of Electrical Engineering and \\ Research Centre for Intelligent Machines \\ McGill University \\ Montréal, Québec, H3A 2A7, Canada \\ grant@cim.mcgill.ca, hayward@cim.mcgill.ca
}

\begin{abstract}
Further development of a three level switching controller [3] is presented. The controller was originally designed to drive a novel Shape Memory Alloy (SMA) actuator consisting of a number of thin NiTi fibers woven in a counter rotating helical pattern around supporting disks. While the original controller performed satisfactorily, it was hampered by the presence of limit cycles at higher gains. By allowing the upper switching level to be proportional to the velocity it is possible to achieve a damped response, analogous to $P D$ control for linear systems, effectively quenching the limit cycle. With this damping, it is possible to decrease the rise time by almost half and maintain the same steady state accuracy.
\end{abstract}

\section{Introduction}

A considerable amount of work has been concerned with the modelling of shape memory alloy actuators [1], [5], [6], while relatively less attention has been paid to the design of feedback control laws. Many feedback control techniques reported in the literature applied to shape memory actuators are in fact linear compensators such as P, PD or PID controllers [6], [7].

The dynamics of shape memory actuators are predominantly nonlinear since the energy conversion principle, from heat to mechanical, relies on exploiting phase transitions in a metal. This creates at least significant hysteresis in addition to many other nonlinear effects with or without memory. In addition, the detailed properties of the dynamics of shape memory alloys vary widely with their metallurgy content, fabrication process, training techniques [4], aging, and ambient conditions, thus a great deal of parametric uncertainty is involved. Further, most of the detailed descriptions of their underlying physics are often not helpful in controller design, and in the case of linear controllers one usually ends up with an overly conservative design.

Several nonlinear control techniques are also available. However several were immediately discarded due to the large uncertainty in plant parameters, such as those relying on precise nonlinear identification, adaption and feedback linearization. Using describing functions was also ruled out as this would again result in an overly conservative controller. This naturally led to the use of robust variable structure control.

\subsection{Variable Structure Control}

The advantage of variable structure control is that relatively few parameters representing the physical properties of the plant need to be known, since only inequality conditions need to be satisfied in the design [8]. It is also well known that variable structure control is quite insensitive to plant parameter variations since the resulting trajectory resembles a time near-optimal switching curve.

It is often stated that a disadvantage of variable structure control is the discontinuous nature of the control signal which may cause problems in terms of ringing, excessive dissipation, and excitation of unwanted dynamics. For some actuation techniques, switching is not a problem and clearly shape memory alloy actuation is one of them: the mechanical energy is derived from heat which makes the actuators naturally low pass and thus the higher order dynamics are undisturbed by step or impulse inputs. Moreover, the robustness properties of variable struc- 
ture control combined with the modelling difficulties of shape memory alloy actuators creates considerable incentive to apply the former to the latter.

\section{Background}

This paper expands on the variable structure control of the Shape Memory Alloy actuator described in [2]. Recall that the actuator, shown in Figure 1, consists of twelve 100 micron diameter NiTi fibers woven in a counter rotating helical pattern around supporting disks. The disks are separated by preloading springs that keep the fibers under tension when relaxed. When the fibers are heated, they contract pulling the disks together.

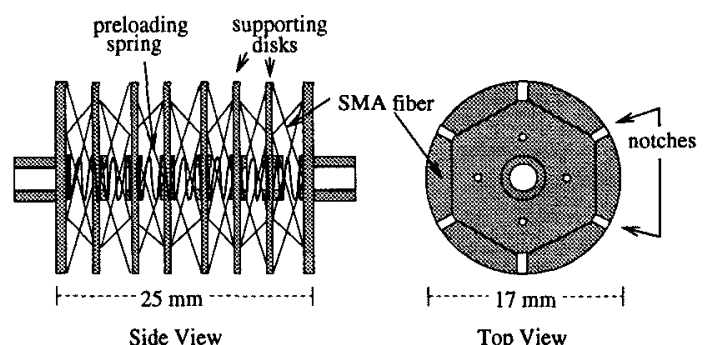

Figure 1: Shape memory alloy actuator

The weave pattern of the fibers accomplishes a displacement amplification that overcomes the main drawback of shape memory alloy devices, that being limited strain.

A three stage switching controller was applied to the actuator [3]. The feedback consisted of three constant magnitude pulses applied to an antagonistic pair of actuators as shown in Figure 2,

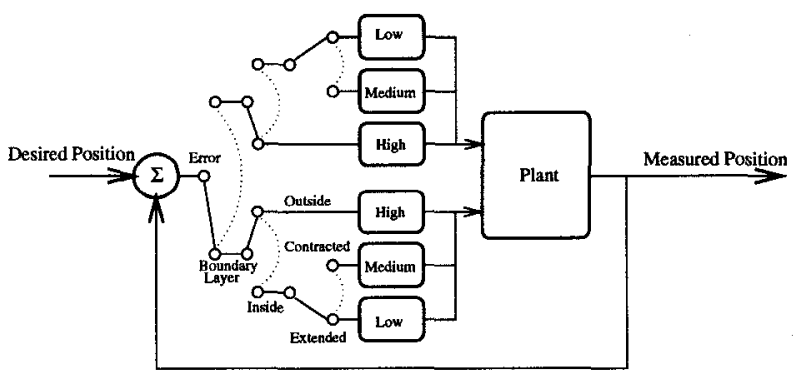

Figure 2: Block diagram of refined controller

The feedback switches between the two actuators according to the sign of the displacement error. The magnitude of the feedback depends on the size of the error and whether the actuator is extended or contracted. This controller was designed for simplicity and speed of response. High gains were used to achieve rapid response, while dithering the plant around the set point, allowing a small average steady state error.

\section{Controller Design}

\subsection{Basic Controller}

The basic controller described in the previous section was designed for rapid response, and therefore high gains were employed. The design variables in the controller are the magnitudes of the constant current pulses (designated High, Med, and Low or $I_{H}, I_{M}$ and $I_{L}$ ) and the width of the boundary layer (designated $B w$ ) around the switching surface as shown in Figure 3 .

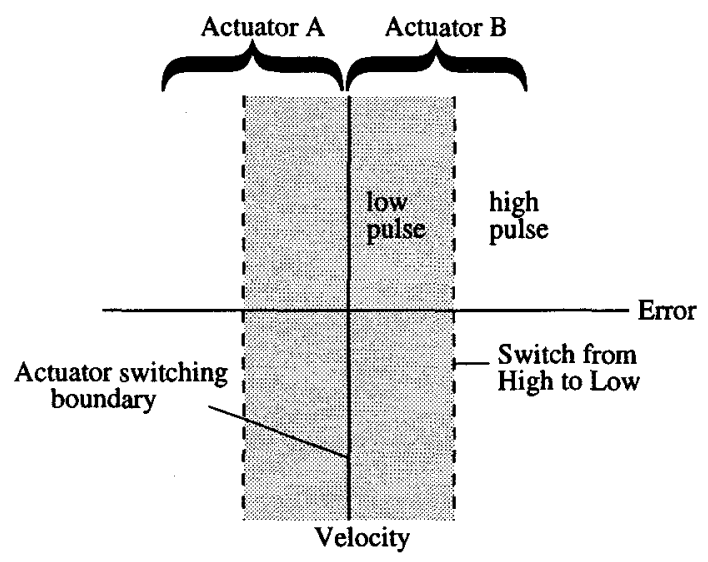

Figure 3: Adjustable Parameters in 3-Stage Controller

Using only one magnitude pulse it was not possible to realize the combined goals of stability and speed of response, therefore a boundary layer was used around the set point. Inside the boundary layer the controller feeds back the $I_{M}$ or $I_{L}$ pulse, depending on whether the actuator is extended or contracted. This is to partially counteract the nonlinearity in the actuator's kinematic gain. Whether the gain is $I_{M}$ or $I_{L}$ inside the boundary layer is not relevant to this discussion and both pulses will be labelled as $I_{L}$ from here on.

Sacrificing time performance and using lower gains with the basic controller produces satisfactory results. However it is difficult to tune the boundary layer width to achieve zero steady state error for a wide range of step responses. In Figure 4 several different magnitude 
step responses can be seen of a controller set with: $I_{H}$ $=3.6 \mathrm{~A}, I_{L}=1.5 \mathrm{~A}$, and $B w=.10 \mathrm{~mm}$. Note that the fibers in the actuator are connected electrically in parallel, so that the current in the individual fibers is divided by a factor of twelve. The antagonistic actuator pair drives an inertial load of $200 \mathrm{~g}$ with some static friction.

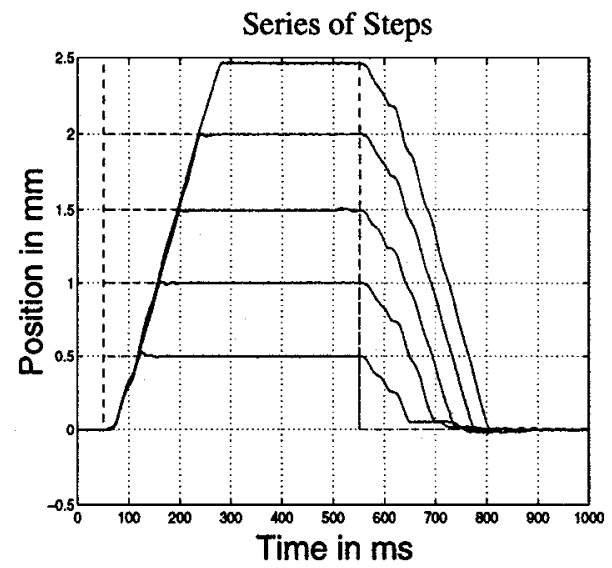

Figure 4: Step Response of Low Gain Controller

From Figure 4 it can be seen that with the low gains the plant does not overshoot the desired position. The steady state error here is also virtually zero except for the $2.5 \mathrm{~mm}$ pulse where there exists a steady state error of $.038 \mathrm{~mm}$. Aside from time performance penalties, another disadvantage of using low gains for the SMA actuator is a lack of robustness to disturbances.

\subsection{Limit Cycles}

Maintaining the same boundary width, and increasing the $I_{H}$ gain we begin to see a more oscillatory response and finally the presence of a limit cycle, as seen in Figure 5.

As the gain increases the peak velocity of the actuator increases, and the more momentum the actuator has when it switches from the high to low pulse. If the velocity is sufficiently bounded or the boundary layer is sufficiently wide, the state trajectory will not cross the actuator switching surface, and will then approach the set point, as shown in the phase plot of Figure 6 .

If, however the gain is large enough, the state trajectory will travel past the switching surface, causing the other antagonistic actuator to turn on, and drive the system the other way. These oscillations will diminish to the origin if the initial crossing velocity is not high enough to cause the state trajectory to pass through the boundary layer, as shown in Figure 7.

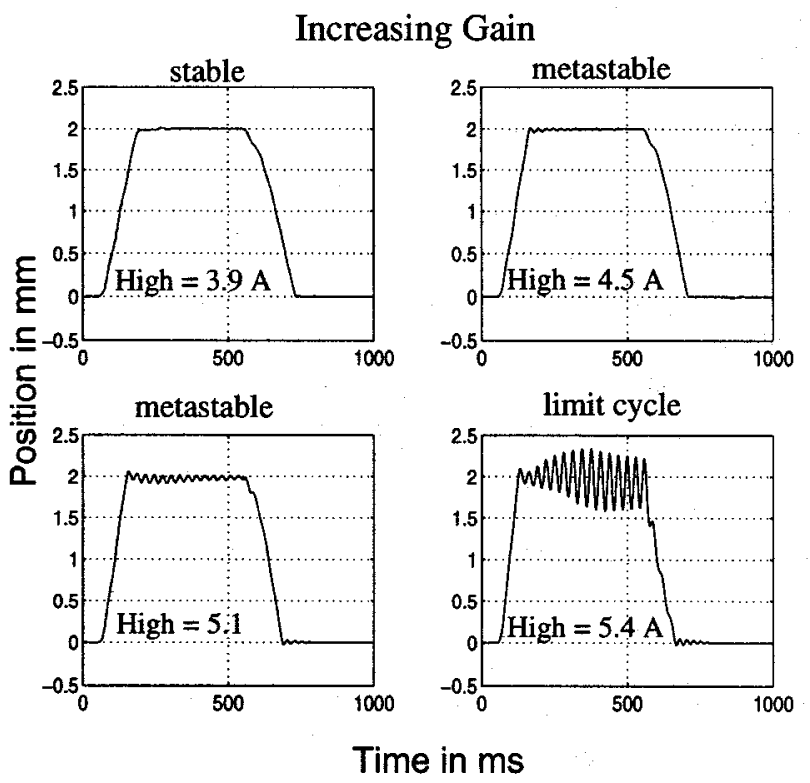

Figure 5: Stable, Metastable, and Limit Cycles

Phase Plot

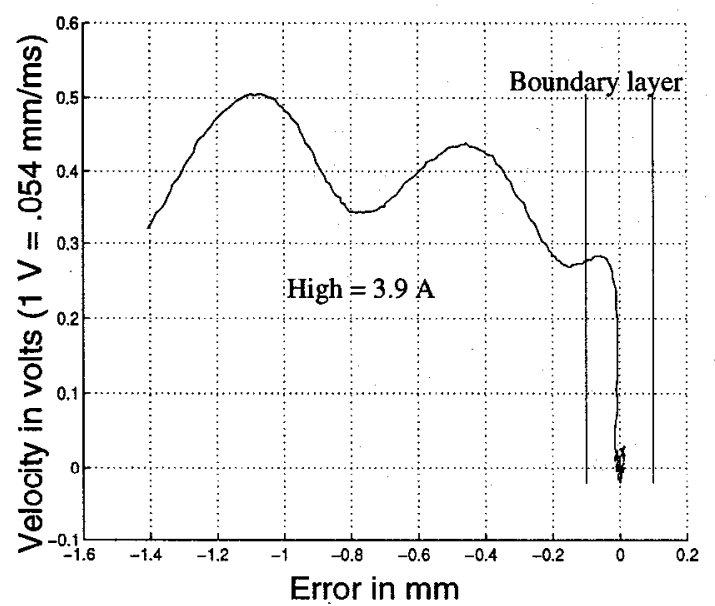

Figure 6: Low Gain 
Due to a lag in the velocity measurement, the phase plot trajectories are tilted to the right.

\section{Phase Plot}

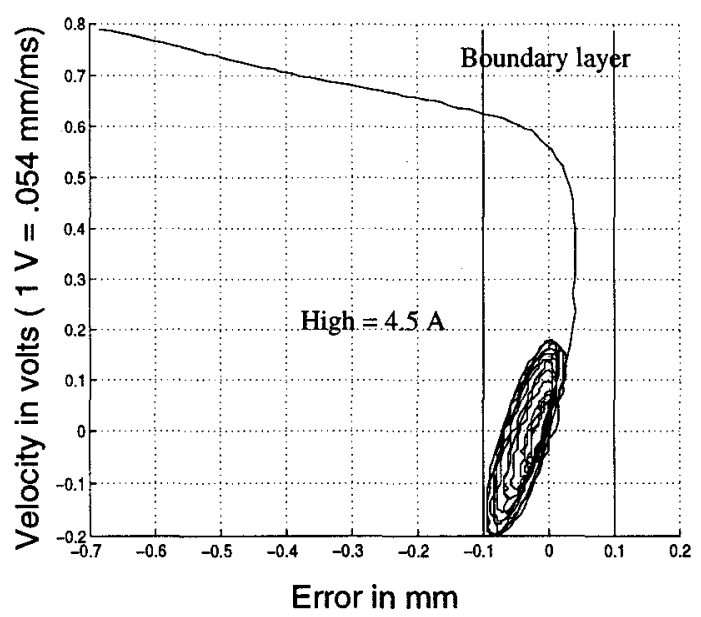

Figure 7: Convergence to the Origin

A stable attractor limit cycle with a large amplitude will result if the actuator has enough momentum to cross through the boundary layer, activating the $I_{H}$ gain of the antagonistic actuator, as shown in Figure 8.

Phase Plot

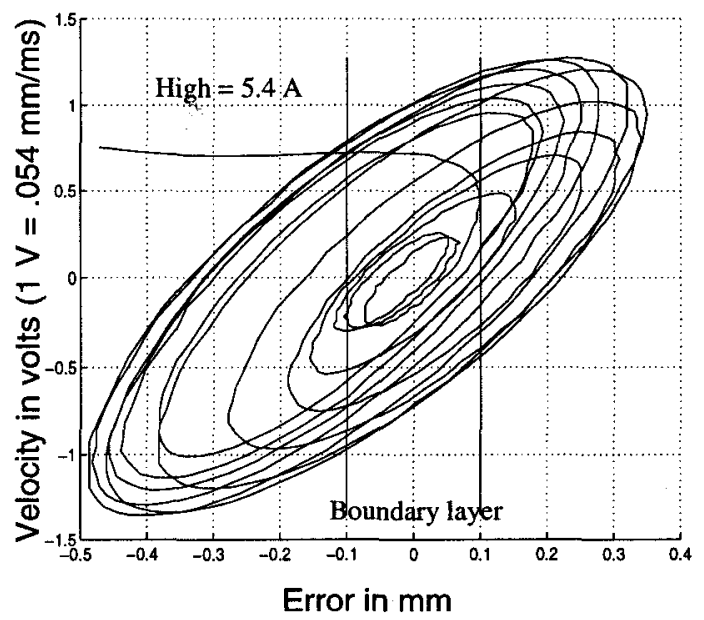

Figure 8: Stable Limit Cycle

\subsection{Damping}

Limit cycles are often undesirable in robotic applications as they cause excitation of higher order dynamics which can lead to instabilities, and excessive wear of mechanical components. From examining the phase plots of the actuator's response with increasing gain, it can be seen that in order to maintain stable behaviour it is necessary to insure that the actuator's velocity is bounded when it crosses the actuator switching boundary. One simple way to accomplish this is to increase the boundary width, causing the actuator to slow down further from the set point. However, the gains inside the boundary layer are chosen to be small for stability, and the more we increase the width, the slower the overall response will be. Ideally we would like to increase the boundary layer width in proportion with the velocity as shown in Figure 9.

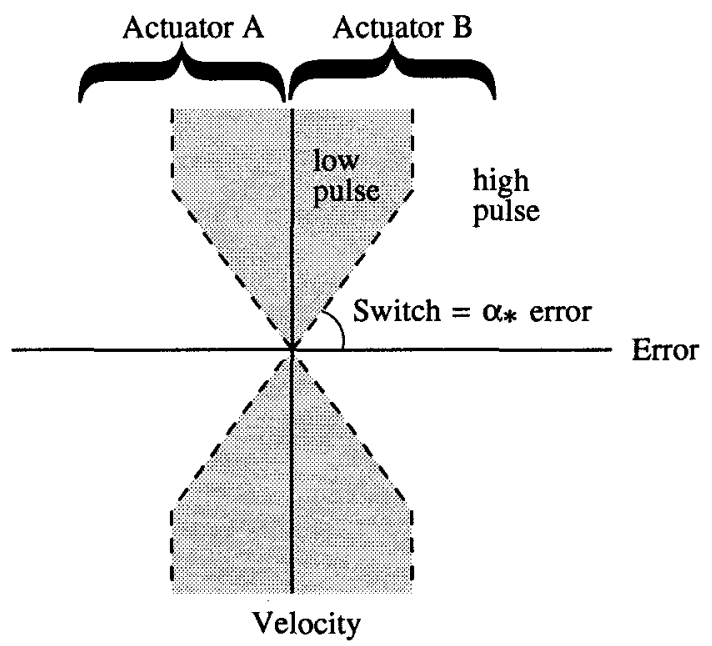

Figure 9: Adjustable parameters for Damping

Increasing the boundary width when the actuator velocity is high allows the system more time to slow down as it approaches the set point. This prevents the state trajectory from crossing the switching surface with enough momentum to create unnecessary oscillations or limit cycles.

When the system is far from the desired position, we require maximum gain, hence the boundary width reaches a maximum at a chosen error bound. As $\alpha$ increases, the $I_{L}$ gain is fed back to the plant for a longer time, effectively increasing the damping. With this controller it will be possible to increase the $I_{H}$ gain, thereby increasing the time domain performance, without creating limit cycles.

\section{Experimental Results}

With the $I_{H}$ gain set to $5.4 \mathrm{~A}$, the basic controller enters a limit cycle, as can be seen in Figure 5. The 


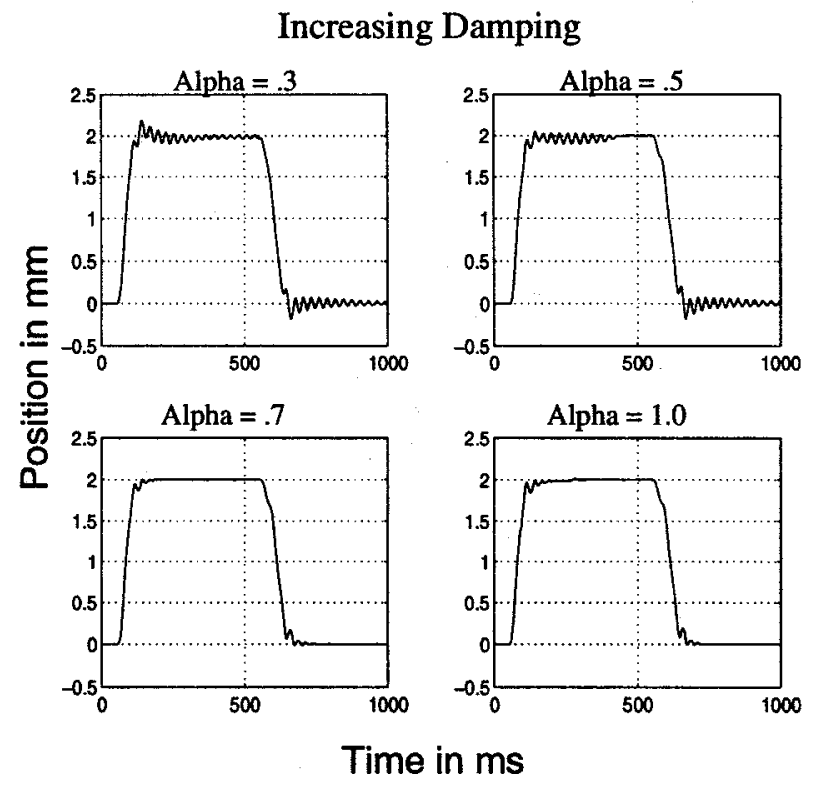

Figure 10: Increasing Damping

When $\alpha$ is low the response is oscillatory, but does not enter a limit cycle. Figure 11 shows the phase plot of the step response when $\alpha=0.5$.

A dead band was inserted for the physical implementation of the controller to avoid switching on the noise. The system here does not enter a limit cycle as the upper switching surface of the antagonistic actuator is tilted away from the state trajectory. The response however is still oscillatory, as the system cycles between the $I_{H}$ and $I_{L}$ pulses for one of the actuators.

As $\alpha$ is increased, the response becomes more and more damped, as can be seen in the phase plot of Figure 12.

With $\alpha=1.0$, the state trajectory does not cross the actuator switching level until the velocity is reduced, preventing any unnecessary oscillations. The final controller has zero overshoot and a steady state error within the limits of the sensors, $(.004 \mathrm{~mm})$. Also, the rise time over the basic controller has decreased by almost a half, from $90 \mathrm{~ms}$ to $52 \mathrm{~ms}$, as can be seen in Figure 13.

The slight ringing that is present is a result of having only two magnitudes of feedback present. When switching from the $I_{H}$ to $I_{L}$ pulse there is quite a difference in applied energy. This ringing can easily be smoothed out if necessary by the addition of another fixed magnitude pulse between the $I_{H}$ and $I_{L}$ pulses.
Phase Plot

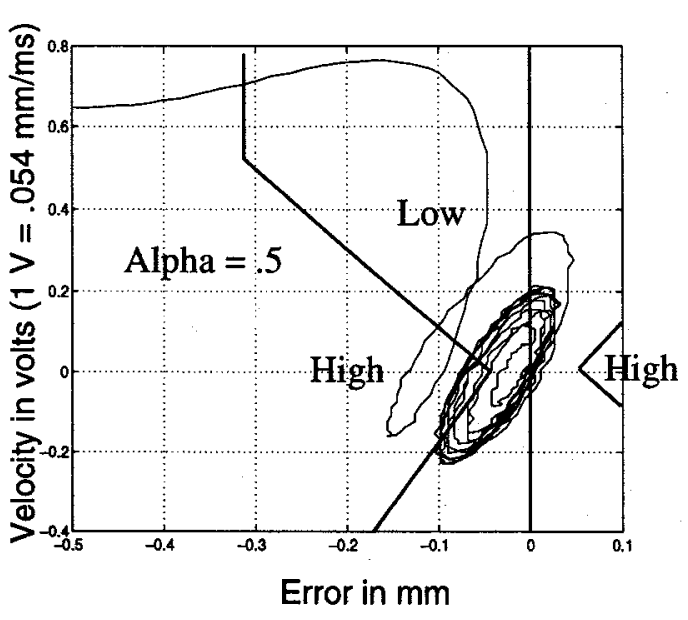

Figure 11: Phase plot : $\alpha=.5 *$ error

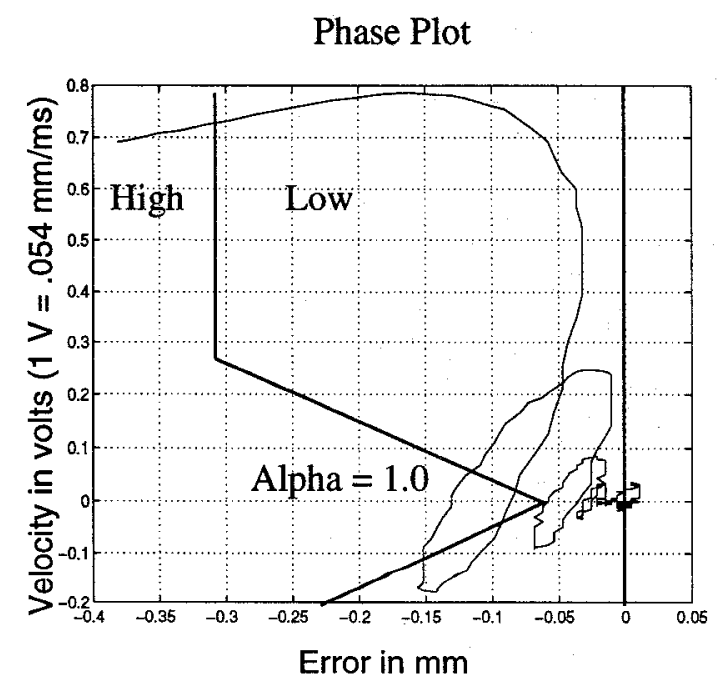

Figure 12: Phase plot : $\alpha=1.0 *$ error 


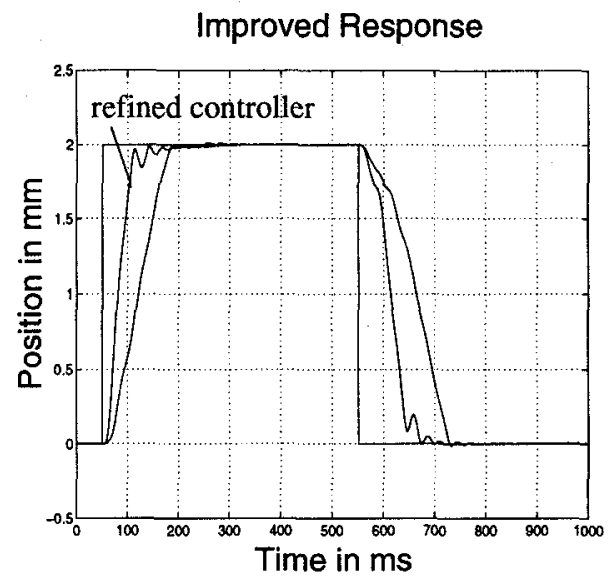

Figure 13: Improved Response

\section{Conclusions}

Introducing a boundary width that is dependent on the velocity allows us to realize the combined goals of rapid response and accuracy. In examining Figure 14 we see that it is possible to completely eliminate the presence of a limit cycle while retaining the benefits of using a higher gain.

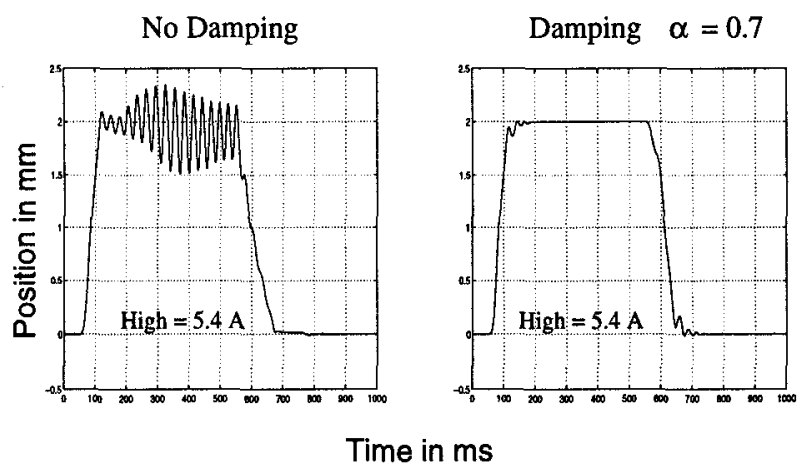

Figure 14: Step Response with and without Damping

This is analogous to adding damping to a linear proportional controller in that it allows us to increase the gain, while maintaining stable performance. The higher gains allowed by the damped controller also provide for a more robust system with better disturbance rejection. Current research is focused on more rigorous proofs of stability for these controllers.

Acknowledgements The research was mostly funded by IRIS (Phase II), the Institute for Robotics and Intelligent Systems part of Canada's National
Centers of Excellence program (NCE), under the heading "Machine Sensing and Actuation: Computational Sensing for Vision and Robotics" (MSA-4), and an operating grant from NSERC, the National Science and Engineering Council of Canada.

\section{References}

[1] D.Yang, G.Lin, and R.Warrington. A computational model of shape memory alloys for the design and control of micro-actuators. Micromechanical Systems, v4, 1992.

[2] D. Grant. Shape memory alloy actuator with an application to a robotic eye. Master's thesis, McGill University, 1995.

[3] D. Grant and V. Hayward. Design of shape memory alloy actuator with high strain and variable structure control. In Proceedings of 1995 IEEE International Conference on Robotics and Automation, volume 3, pages 344-350, Nagoya,Aichi,Japan, May 21-27 1995.

[4] I. Hunter, S. Lafontaine, J. Hollerbach, and P. Hunter. Fast reversible NiTi fibers for use in microrobotics. In Microelectro-mechanical Systems, Nara,Japan, Jan 1991.

[5] K. Ikuta, M. Tsukamoto, and S. Hirose. Mathematical model and experimental verification of shape memory alloy for designing micro actuator. In Proc. of the IEEE MicroElectroMechanical Systems Conference, pages 103-108, 1991.

[6] D. R. Madill. Modelling and stability of a shape memory alloy position control system. Master's thesis, Applied Science, University of Waterloo, 1993.

[7] D. Reynaerts and H. Van Brussel. Development of a SMA high performance robotic actuator. In Fifth International Conference on Advanced Robotics, volume 2, pages 19-27, New York, NY, 1991.

[8] V. L. Utkin. Sliding Modes in Control Optimization. Springer-Verlag, 1992. 